\title{
Intraadiposal Pedicle Dissection when Performing Free Tissue Transfer: A Means to Address a Short Pedicle
}

\author{
Jung Han Lim (D), Jung Woo Chang (D) \\ Department of Plastic and Reconstructive Surgery, Hanyang University Guri Hospital, Hanyang University College of Medicine, Guri, Korea
}

\begin{abstract}
A 77-year-old male patient with a diabetic foot ulcer on his right great toe visited the outpatient clinic. As necrosis of the toe had already progressed, the patient underwent toe amputation to prevent extensive gangrene. With the goal of enabling future ambulation, an anterolateral thigh fasciocutaneous free flap was planned to preserve the metatarsal head and to cover the defect. Subfascial dissection was performed when elevating the fasciocutaneous flap, but the sole healthy perforator was in an extremely proximal area and allowed only a 4-cm-long pedicle. The pedicle had to be at least $8 \mathrm{~cm}$ long to ensure secure anastomosis to the medial plantar artery. To overcome this unexpected challenge, we pierced the deep fascia near the perforator and dissected the perforator distally within the deep adipose layer. The necessary additional length of the pedicle was obtained through intraadiposal dissection. The flap survived without any complications, and the foot was reconstructed with successful ambulation. This case demonstrates the value of attempting intraadiposal pedicle dissection when the pedicle turns out to be unmanageably short.
\end{abstract}

Keywords: Diabetic foot; Perforator flap; Free flap

\section{Introduction}

Diabetic foot ulcers are slow to heal because of chronic tissue ischemia, distal neuropathy, and increased tissue glucose levels, all of which lead to abnormal cellular function [1]. Accompanied by peripheral artery disease, these chronic wounds contribute to many major amputations. Recently, the use of microvascular free flaps has increased the percentage of limbs salvaged, contributing to improvements in quality of life and prospects of survival $[2,3]$. However, surgeons sometimes face unanticipated situations when elevating a flap in a diabetic foot, such as the lack of a proper recipient vessel due to atherosclerotic and obstructive changes and a relatively short pedicle in the flap. When the distal recipient vessel is in poor condition, and particularly when the defect is located on the distal foot, a longer vascular pedicle is required to use a more proximal recipient vessel. Furthermore, if the flap has only a short pedicle with other perforators not suitable for use, the vascular pedicle may not be long enough for safe anastomosis. These situations, which make free flaps in diabetic foot more challenging, underscore the importance of considering a variety of methods to achieve longer pedicles. Though a vein graft is a possible option to resolve this problem, it requires further donor morbidity for vein harvesting and additional microanastomosis, which is time-consuming $[4,5]$.

In this case report, we present a case of diabetic foot with an unexpectedly short
Case Report

Received: December 29, 2020

Revised: February 2, 2021

Accepted: February 3, 2021

\section{Corresponding author:}

Jung Woo Chang, M.D., Ph.D.

Department of Plastic and Reconstructive Surgery, Hanyang University Guri Hospital, 153 Gyeongchun-ro, Guri 11923, Korea Tel: +82-31-560-2330

Fax: +82-31-560-2338

E-mail: mickey404@hanmail.net

This is an Open Access article distributed under the terms of the Creative Commons Attribution Non-Commercial License (https://creativecommons org/licenses/by-nc/4 . O/) which permits unrestricted non-commercial use, distribution, and reproduction in any medium, provided the original work is properly cited.

C) 2021 Korean Wound Management Society 
pedicle. An intraadiposal pedicle dissection was performed to address the situation. This study was conducted in conformance with the Declaration of Helsinki of the World Medical Association. The patient provided written informed consent for the publication and the use of his images.

\section{Case}

A 77-year-old male patient with a diabetic foot ulcer on his right great toe visited the outpatient clinic of the department of plastic and reconstructive surgery. Toe skin necrosis and osteomyelitis of the phalangeal bones had already progressed. To prevent progression to extensive gangrene, amputation of the toe was planned. The patient underwent disarticulation at the metatarsophalangeal joint level and complete debridement (Fig. 1A). Though the usual practice is to sacrifice the metatarsal head of the great toe to achieve primary closure of the defect wound, we planned an anterolateral thigh fasciocutaneous free flap to preserve the metatarsal head and to cover the defect, with the goal of enabling future ambulation.

Computed tomography angiography of lower extremities was performed preoperatively to evaluate the vascular condition. The image showed poor vascularity of the anterior tibial artery with total occlusion of dorsalis pedis artery and first
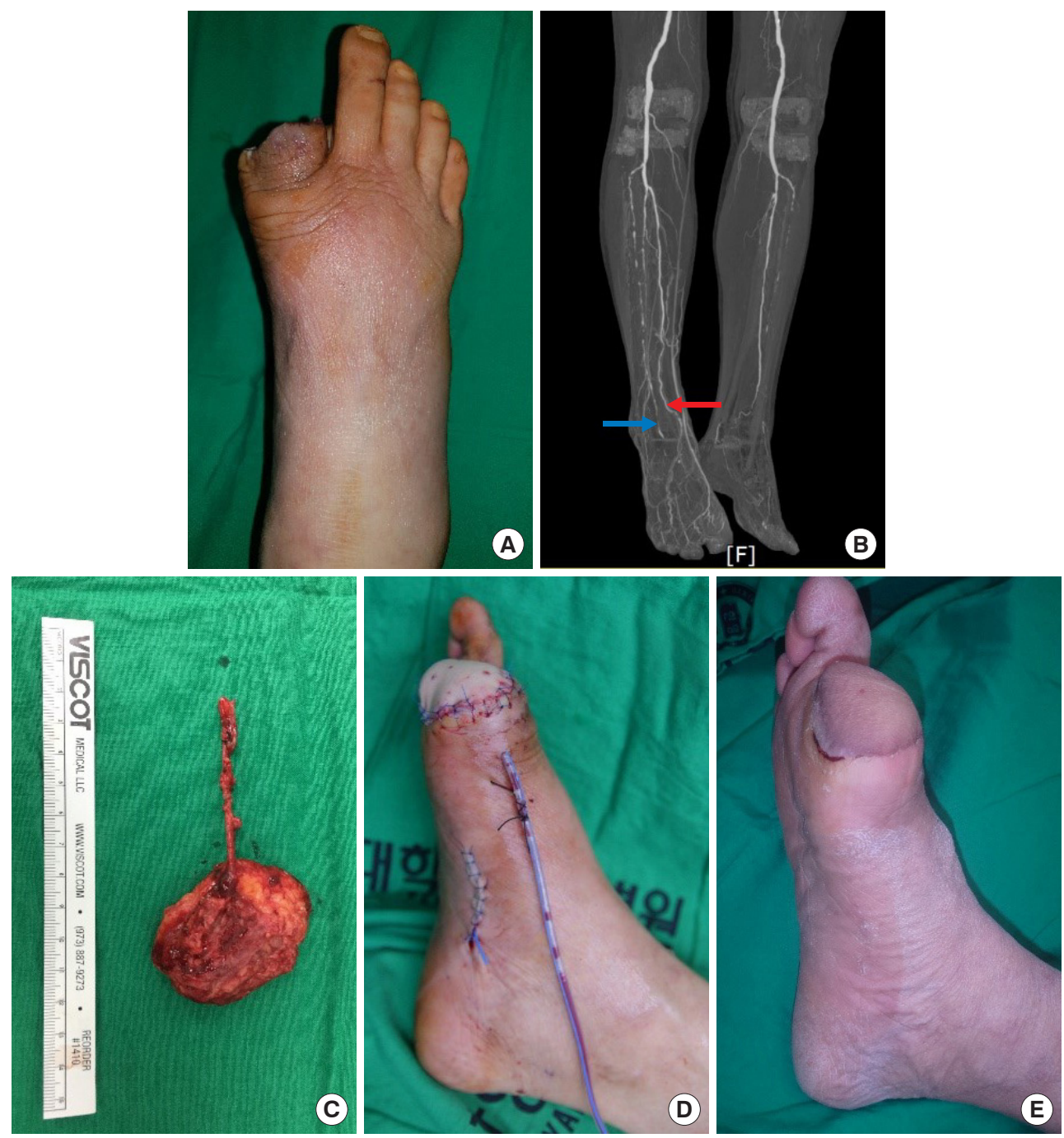

Fig. 1. Perioperative findings of diabetic foot reconstruction case. (A) A 77-year-old male patient with a diabetic foot ulcer on his right first toe underwent toe amputation and complete debridement. (B) Steno-occlusion of anterior tibial artery (blue arrow) and patent posterior tibial artery (red arrow) of right foot. (C) In a free anterolateral thigh perforator flap, additional pedicle length was achieved through intraadiposal dissection. (D) A secure anastomosis was made to the medial plantar artery. (E) At a 6-month postoperative follow-up visit, the flap had stabilized without any complications. 
dorsal metatarsal artery (Fig. 1B). The posterior tibial artery, on the other hand, seemed relatively patent. Intraoperatively, the medial plantar artery, about $8 \mathrm{~cm}$ from the defect, was found to be the nearest patent vessel. We therefore decided to use the medial plantar artery as the recipient vessel. The distal part of the vessel, in poor condition due to atherosclerotic changes, was removed and the appropriate recipient vessel was exposed. The distance from the defect to the healthy recipient vessel was again approximately $8 \mathrm{~cm}$. Subfascial dissection was performed to elevate the fasciocutaneous flap.

However, the vascular condition of the donor site was poorer than expected; the only healthy perforator was in an extremely proximal area and allowed only a $4-\mathrm{cm}$-long pedicle. For secure anastomosis to the medial plantar artery, the pedicle had to be at least $8 \mathrm{~cm}$ long. To overcome this problem, before ligating the proximal part of the main pedicle, we pierced the deep fascia near the perforator and dissected the perforator distally within the deep adipose layer. The deep adipose layer along the pedicle was meticulously dissected with sharp mosquito forceps until the superficial fascia was identified. An additional $4 \mathrm{~cm}$ of pedicle length could be obtained through this procedure and the flap was moved slightly distally without difficulty. We elevated a $5.5 \times 4.5-\mathrm{cm}$ suprafascial flap with a longer pedicle, allowing secure anastomosis (Fig. 1C and D). The flap survived without any complications (Fig. 1E). During long-term follow-up, the patient successfully regained ambulation. Free tissue transfer was indeed an appropriate treatment, considering the patient's postoperative quality of life.

\section{Discussion}

As current data show that diabetic foot reconstruction improves the limb salvage rate, quality of life, and potential for survival, the diabetic foot is no longer a contraindication to free tissue transfer [3]. In free flap surgery, obtaining an appropriate length of pedicle is highly important for successful vascular anastomosis, as it determines the tension of the anastomosis site [6]. In addition to the unpredictable length of the pedicle in free flap surgery, poor conditions of the recipient vessel in diabetic foot reconstruction increases the risk of anastomosis failure if the pedicle is insufficiently long. Surgeons have explored ways to solve this problem.

The course of the perforator artery in an anterolateral thigh flap typically derives from the descending branch of the lateral circumflex femoral artery, runs downward, pierces the deep fascia, and passes through the deep and superficial adipose layer (Fig. 2A). To obtain sufficient length of this perforator artery in a classical fasciocutaneous flap, retrograde dissection of perforator (from distal to proximal) through the intramuscular layer is performed (Fig. 2B). Although the anterolateral thigh flap is known for its potential for a long vascular pedicle, the pedicle may be shorter $(4-8 \mathrm{~cm})$ if it is harvested more proximally [7]. In our case, the vascular condition of the perforators was poorer than expected, and the only healthy perforator was located in an extremely proximal area. The pedicle was not long enough for anastomosis after intramuscular pedicle dissection of the most proximal region. We therefore considered using the hidden length of the pedicle above the deep fascia. If perforators are absent or in poor condition when elevating an anterolateral thigh flap, this is an option for elongating a short pedicle.

When raising a suprafascial flap after intraadiposal dissection, the extended distal part of pedicle can be observed in the deep adipose layer (Fig. 2C). A major advantage of this method is that vein grafting can be avoided. Though vein grafting is
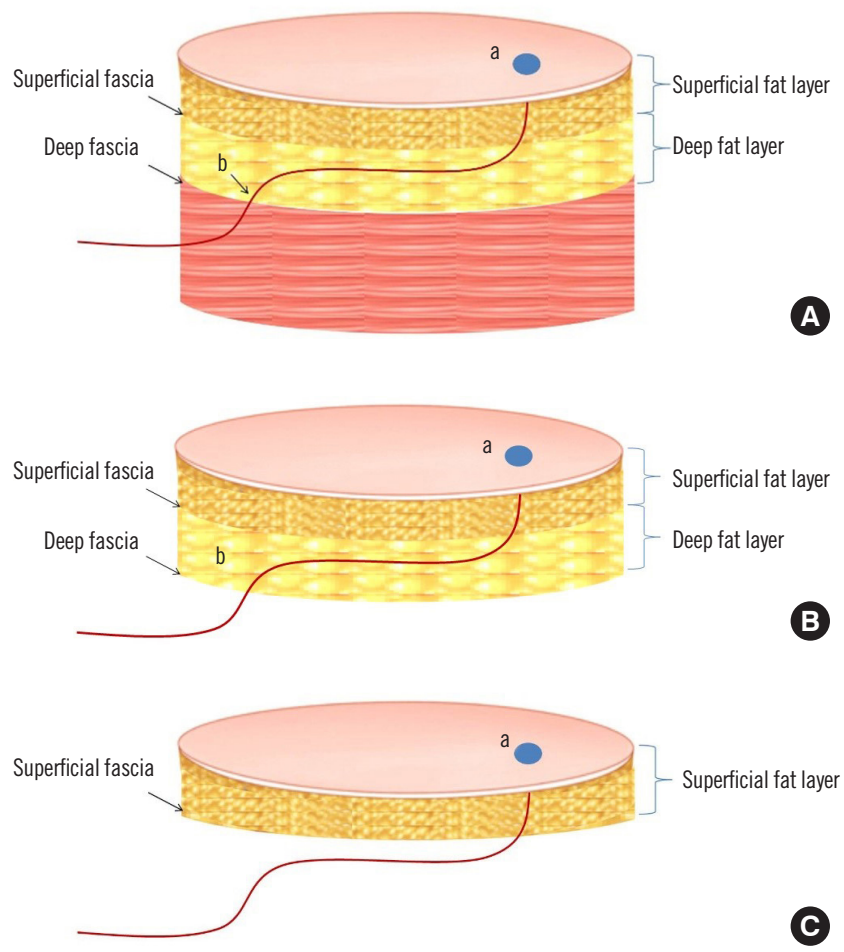

Fig. 2. Schematic image of perforator course in the flap. (A) A schematic image showing the perforator of the flap. (B) The fasciocutaneous flap in the preoperative plan included both deep and superficial adipose layers. (C) After pedicle dissection in the deep adipose layer, a suprafascial flap with a lengthened pedicle was elevated. a, skin marking of Doppler ultrasound tracing; b, point where the perforator pierced the deep fascia. 
considered for cases with a short pedicle [4,5], it has the disadvantage of requiring further donor morbidity for vein harvesting and time-consuming additional micro-anastomosis. Huang et al. [8] reported a case of in situ pedicle lengthening by anastomosis of the proximal part of the perforator, which was the oblique branch of the lateral circumflex femoral artery, to the most distal part of the descending branch of the artery. This method offers pedicle elongation without consuming extra flap ischemic time, but it requires additional micro-anastomosis. In contrast, intraadiposal pedicle dissection is a relatively simple, time-efficient, and immediately available way to obtain additional pedicle length.

While thin suprafascial flaps may seem unsuitable because of their insufficient vascularity, Maruccia et al. [9] and Hong and Chung [10] reported cases of extremely thin anterolateral thigh free flaps that were successful thanks to dominant vascular connections occurring within the dense subdermal plexus. Saint-Cyr et al. [11] proposed the perforasome theory, which describes the interconnection of perforators that are mainly located in the suprafascial and subcutaneous layers and the subdermal plexus. These interconnections explain the adequate perfusion of suprafascial anterolateral thigh flaps. In addition to being functional without the drawbacks of a bulkier flap, the suprafascial anterolateral thigh flap is hemodynamically reliable and safe $[10,12]$. It should be noted, however, that the thinning procedure employed in this case differed from that of conventional suprafascial flaps. When elevating a suprafascial flap, a retrograde dissection (from distal to proximal) of the perforator is preferred as it is known to be safer than the anterograde dissection performed in this case. However, in this case, we had preoperatively planned a fasciocutaneous flap instead of a suprafascial flap and initially detected the perforator under the deep fascia. Anterograde perforator dissection is inevitable for such unplanned suprafascial flaps, even if it is considered less safe than a retrograde procedure.

A shortcoming of this method is that the length of the hidden pedicle and the course of the perforator in the deep adipose layer is unpredictable. Such information is not available neither preoperatively nor even intraoperatively, unless an intraadiposal pedicle dissection is performed. Therefore, there is the risk that the hidden pedicle may not be sufficiently long.

Although we cannot predict the accurate course and length of the pedicle in the adipose layer, our case suggests that in certain cases, intraadiposal pedicle dissection is still an option worth attempting. It may not be a perfect alternative when the pedicle is found to be too short, but it is nevertheless an option that can be tried before considering other possibilities, such as vein grafting.

\section{Conflict of interest}

Jung Woo Chang is an editorial board member (Managing Editor) of the journal but was not involved in the peer reviewer selection, evaluation, or decision process of this article. No other potential conflicts of interest relevant to this article were reported.

\section{ORCID iDs}

Jung Han Lim

https://orcid.org/0000-0002-0202-3674

Jung Woo Chang

https://orcid.org/0000-0002-7937-9679

\section{References}

1. Game FL, Jeffcoate WJ. Dressing and diabetic foot ulcers: a current review of the evidence. Plast Reconstr Surg 2016; 138(3 Suppl):158S-164S.

2. Sato T, Yana Y, Ichioka S. Free flap reconstruction for diabetic foot limb salvage. J Plast Surg Hand Surg 2017;51: 399-404.

3. Fitzgerald O'Connor EJ, Vesely M, Holt PJ, et al. A systematic review of free tissue transfer in the management of non-traumatic lower extremity wounds in patients with diabetes. Eur J Vasc Endovasc Surg 2011;41:391-9.

4. Inbal A, Silva AK, Humphries LS, et al. Bridging the gap: a 20-year experience with vein grafts for free flap reconstruction. The odds for success. Plast Reconstr Surg 2018;142: 786-94.

5. Bayramicli M, Tetik C, Sonmez A, et al. Reliability of primary vein grafts in lower extremity free tissue transfers. Ann Plast Surg 2002;48:21-9.

6. Ishii N, Kishi K. Determining length and position of pedicle vessels using a flap model with vascular tape. Plast Reconstr Surg Glob Open 2016;4:e1161.

7. Lakhiani C, Lee MR, Saint-Cyr M. Vascular anatomy of the anterolateral thigh flap: a systematic review. Plast Reconstr Surg 2012;130:1254-68.

8. Huang YH, Hsieh TY, Lai CS, et al. In situ pedicle lengthening of the anterolateral thigh flap. Plast Reconstr Surg 2014;133:85e-87e.

9. Maruccia M, Fallico N, Cigna E, et al. Suprafascial versus traditional harvesting technique for free antero lateral 
thigh flap: a case-control study to assess the best functional and aesthetic result in extremity reconstruction. Microsurgery 2017;37:851-7.

10. Hong JP, Chung IW. The superficial fascia as a new plane of elevation for anterolateral thigh flaps. Ann Plast Surg 2013;70:192-5.

11. Saint-Cyr M, Wong C, Schaverien M, et al. The perfora- some theory: vascular anatomy and clinical implications. Plast Reconstr Surg 2009;124:1529-44.

12. Chen YC, Scaglioni MF, Carrillo Jimenez LE, et al. Suprafascial anterolateral thigh flap harvest: a better way to minimize donor-site morbidity in head and neck reconstruction. Plast Reconstr Surg 2016;138:689-98. 